\title{
Medical Image Fusion Combined with Accelerated Non-negative Matrix Factorization and Expanded Laplacian Energy in Shearlet Domain
}

\author{
Siyu Lai ${ }^{1}$, Juan Wang ${ }^{2 *}$, Chunlin $\mathrm{He}^{2}$ and Thanos Hannah Borjer ${ }^{3}$ \\ ${ }^{1}$ Department of Medical Imaging, North Sichuan Medical College, Nanchong 637000, China \\ ${ }^{2}$ College of Computer Science, China West Normal University, Nanchong 637002, China \\ ${ }^{3}$ Information Institute for Geographic Engineering, ABB Communication Corporation, Patras 138682, Greece
}

Received 20 February 2017; Accepted 11 August 2017

\begin{abstract}
Shearlet transform has been widely applied in related fields due to its admirable properties in image approximation. Image fusion method based on accelerated non-negative matrix factorization (ANMF) and expanded energy of Laplace (EEOL) rules was proposed in this study to integrate the complementary information of medical images with multiple modalities and improve the accuracy of clinical diagnosis and therapy. First, the registered medical images were decomposed into low- and high-frequency sub-band coefficients in shearlet domain. Then, the ANMF rule was used in merging low-frequency coefficients. Next, the visual-contrast-based EEOL rule was adopted in extracting details of source images from high-frequency coefficients. Finally, the ultimate fused image was reconstructed by applying inverse shearlet transform. Experimental results reveal that aside from visual effect, the proposed method achieves the best in three of five criteria and the run time is reduced by $29.21 \%$ compared with a method based on non-subsampled contourlet transform (NSCT) in computed tomography (CT)-magnetic resonance imaging (MRI) fusion. Moreover, the proposed method takes the first place in four of five criteria with run time reduced by $48.32 \%$ and $24.55 \%$ compared with two shearlet-based methods in a MRI-positron emission tomography (PET) case. This study indicates that the proposed method is superior to the selected approaches in visual and statistical evaluation, which is conducive to clinical practice of medical image fusion.
\end{abstract}

Keywords: Medical image, Image fusion, Shearlet, Non-negative matrix factorization, Expanded energy of Laplace

\section{Introduction}

As a branch of information fusion, medical image fusion aims to address medical problems reflected through images of multiple modalities by integrating information with respect to the human body's similar anatomical position, cells, and organs. Corresponding images are often used for diagnosing or assessing certain human organs or tissues given that different imaging devices employ different sensors or imaging principles. Generally, medical images encompass morphological, structural, and functional images [1]. In clinical practice, morphological images are often used to recognize changes of cell shape, structural images are usually applied in identifying bones or soft tissues, and functional images can provide abundant information on human metabolism. Thus, a fused image, which combines the information of multiple modality medical images, enables accurate localization and recognition for abnormalities and provides effective imaging reference in disease diagnosing and therapy [2][3][4].

In recent years, medical image fusion technology has been widely used in disease diagnosis, defect analysis, historical archiving, and judicial authentication. The framework for fusion, imaging modality, and certain organ

*E-mail address: wjuan0712@126.com

ISSN: 1791-2377 @ 2017 Eastern Macedonia and Thrace Institute of Technology. All rights reserved. doi:10.25103/iestr.104.21 are the three basic elements of studies on medical image fusion [5]. To date, few reports or studies have addressed the optimal combination of the aforementioned three factors due to the physical, even mental, differences for observers and diverse mathematical framework. Hence, developing an optimal fusion framework is difficult for a specific image modality or organ.

Based on the above analysis, this study discusses the process of medical image fusion under shearlet framework, in which two improved rules for coefficients fusion are adopted to optimize image quality and time consumption. Subsequently, the validity of the proposed method is evaluated and advantages are summarized.

\section{State of the art}

To date, domestic and foreign scholars have investigated medical image fusion, which is considered to be composed of the space-based and multiscale-based methods. The former has a simple structure to calculate and has been regarded as the mainstream of medical image fusion in earlier stage. Space-based methods include weighted linear, principal component analysis (PCA), multiplicative fusion, and neural network. However, space-based method obtains rough result in expressing detailed information of medical images. Therefore, multiscale-based method is gaining popularity in the field of image fusion in recent years. The 
commonly used multiscale-based methods include wavelet transform (WT), contourlet transform, non-subsampled contourlet transform (NSCT), and shearlet transform.

Scholars have conducted considerable research to apply multiscale-based methods in medical image fusion and optimize the performance of algorithms. In 2014, Singh et al. proposed a method based on Daubechies complex wavelets, which was approximately shift invariant and provided phase information; the method has been proven better compared with other methods [6]. However, being the most typical multiscale decomposition tool, wavelets fail to capture linear singularity, continuous edge, and detail of medical images [7][8]. Darwish et al. presented an image fusion system based on contourlet transform and multilevel fuzzy reasoning technique, in which the fuzzy fusion rules were applied to low- and high-frequency coefficient approximation; the results showed the effectivity of the proposed method for applications in brain image fusion [9]. Clearly, contourlet transform can obtain multi-directional high-frequency information and capture the edge detail of images, although it is not shift-invariant. As a result, clear textures and smooth edges are unavailable when positioning contour or sketching region and pseudo-Gibbs distortion may occur [10][11]. Bhateja et al. introduced a two-stage multimodal fusion framework using the combination of stationary WT and NSCT domains for computed tomography (CT), magnetic resonance imaging (MRI), and positron emission tomography (PET) images; they also depicted the visual and quantitative superiority of the obtained fusion results [12]. The down-sampling step is removed in NSCT to avoid drawbacks in contourlets. However, NSCT requires high computational complexity and a considerable amount of time when managing actual medical images, which limits further development [13][14].

In 2008, Guo constructed shearlets based on a special form of synthetic dilation of the affine structure; shearlet is a novel multiscale geometric analysis tool that overcomes wavelets' shortcomings, which is a nearly optimal sparse representation for multi-dimensional function [15]. Shearlets generate mother function by dilations, translations, and rotations with its simplified mathematical structure and associate the multi-resolution analysis with the application $[16][17][18]$. Compared with other multiscale decomposition tools, shearlets not only enjoy similar nonlinear order of error as curvelets do when approximating the optimum but also subdivide layer by layer in frequency domain [19][20]. In addition, both the number of directions for the decomposition and the size of the support basis are unlimited, hence, shearlets have higher efficiency in computation compared with contourlets [21][22]. Zhou et al. evaluated the performance of shearlet-based texture features of breast tumors in ultrasound images with significant results that have outperformed other methods, especially in detail acquisition phase [23]. Geng et al. proposed a fusion method based on shearlets and pulse-coupled neural network (PCNN), which not only extracts a considerable amount of visual information but also avoids the occurrence of artifacts [4]. Wang et al. developed a multimodal medical image fusion method in shift-invariant shearlet transform domain, in which added singularities are preserved and added functional details are transferred [24].

In the practice of medical image fusion, the quality of the fusion result mainly depends on the adopted fusion framework and rules. Based on admirable features of multiresolution analysis, shearlets are used in conducting multiscale and multi-directional decomposition on original medical images. For the low-frequency coefficients accounting for the contour differences of organs or tissues, the accelerated non-negative matrix factorization (ANMF) rule is adopted. Moreover, for the high-frequency coefficients representing regional energy details, the expanded energy of Laplace (EEOL) rule is employed. This study analyzes the operating characteristics of shearlets and the fusion rules, and designates fusion steps of the proposed method based on "ANMF+EEOL" rules for multiple modalities of medical image fusion under shearlets domain, and illustrates the experimental result. Furthermore, this study aims to explore the working mechanism of shearlets precisely, verify the performance of the proposed fusion rules, and offer reference for further development and improvement of medical image fusion.

The remainder of this study is structured as follows. Section 3 discusses the course of fusion, as well as the fusion rules for low- and high-frequency coefficients. Section 4 presents the results of the experiments for multiple modalities of medical image fusion, followed by the conclusions in Section 5.

\section{Methodology}

\subsection{Shearlet transform}

In applied mathematics, shearlets are a multiscale framework, which allows efficient encoding of anisotropic features in multivariate problem classes. Similar to wavelets, shearlets arise from the affine group and allow a unified treatment of the continuum and digital situation leading to faithful implementations. In Reference [25], shearlets are expressed as:

$$
\Psi_{a, s, t}(x)=a^{-3 / 4} \Psi\left(S_{s}^{-1} A_{a}^{-1}(x-t)\right)
$$

where $A_{a}=\left(\begin{array}{cc}a & 0 \\ 0 & \sqrt{a}\end{array}\right), a>0$, represents the anisotropic parabolic scaling matrix used to change the resolution. $S_{s}=\left(\begin{array}{ll}1 & s \\ 0 & 1\end{array}\right), s \in \mathbb{R}$ denotes the shear matrix used as a means to change the orientation. Finally, shearlets $\Psi_{a, s, t}(x)$ can be constructed by translation.

We have $\Psi \in L^{2}\left(\mathbb{R}^{2}\right)$ satisfying the following conditions: For any $\xi=\left(\xi_{1}, \xi_{2}\right) \in \hat{\mathbb{R}}^{2}\left(\xi_{2} \neq 0\right)$, we assume that $\hat{\Psi}(\varepsilon)=$ $\hat{\Psi}\left(\varepsilon_{1}, \varepsilon_{2}\right)=\hat{\Psi}_{1}\left(\varepsilon_{1}\right) \hat{\Psi}_{2}\left(\varepsilon_{2} / \varepsilon_{1}\right)$, where $\hat{\Psi}(\varepsilon)$ denotes the Fourier transform of $\Psi(\varepsilon) . \Psi_{1}$ is the continuous wavelets, $\hat{\Psi}_{1} \in C^{\infty}(\mathbb{R}), \operatorname{supp}_{1} \subset[-2,-1 / 2] \cup[1 / 2,2] \cdot \hat{\Psi}_{2} \in C^{\infty}(\mathbb{R})$, $\operatorname{supp} \Psi_{2} \subset[-2,-1 / 2] \cup[1 / 2,2]$, where $\hat{\Psi}_{2} \geq 0$ and $\left\|\Psi_{2}\right\|=1$ in interval of $(-1,1)$.

We assume that

$$
\sum_{j \geq 0}\left|\hat{\Psi}_{1}\left(2^{-2 j} \omega\right)\right|^{2}=1, \quad\left(|\omega| \geq \frac{1}{8}\right)
$$

$$
\text { For any } j \geq 0 \text {, }
$$

$$
\sum_{l=-2 j}^{2 j-1}\left|\hat{\Psi}_{2}\left(2^{j} \omega-l\right)\right|^{2}=1, \quad(|\omega| \leq 1)
$$


By the supporting conditions of $\hat{\Psi}_{1}$ and $\hat{\Psi}_{2}$, the function $\Psi_{j, l, k}$ has frequency support as follows:

$$
\begin{aligned}
& \operatorname{supp} \hat{\Psi}_{j, l, k}^{(0)} \subset\left\{\left(\xi_{1}, \xi_{2}\right): \xi_{1} \in\left[-2^{2 j-1},-2^{2 j-4}\right] \cup\right. \\
& \left.\left[-2^{2 j-4},-2^{2 j-1}\right],\left|\frac{\xi_{2}}{\xi_{1}}+l 2^{-j}\right| \leq 2^{-j}\right\}
\end{aligned}
$$

That is, each element of $\hat{\Psi}_{j, l, k}$ is supported by a pair of trapezoids, with a size of approximately $2^{2 j} \times 2^{j}$ and aligns along the lines of slope $l 2^{-j}$, as shown in Fig. 1 .

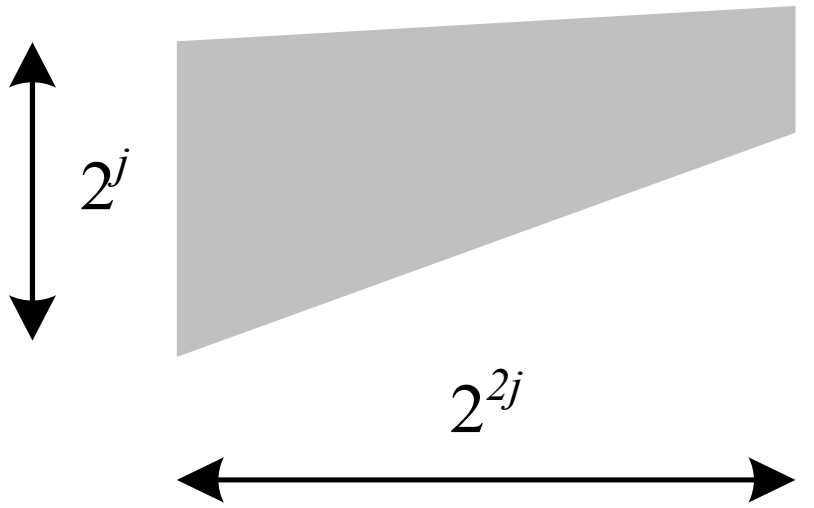

Fig. 1. Trapezoidal frequency support of shearlets

From Eqs. (2) and (3), for any $\left(\xi_{1}, \xi_{2}\right) \in D_{0}=$ $\left\{\left(\xi_{1}, \xi_{2}\right) \in \mathbb{R}^{2}:\left|\xi_{1}\right| \geq \frac{1}{8},\left|\frac{\xi_{2}}{\xi_{1}}\right| \leq 1\right\}$, such that

$\sum_{j \geq 0} \sum_{l=-2 j}^{2 j-1}\left|\hat{\Psi}^{(0)}\left(\xi A_{0}^{-j} B_{0}^{-1}\right)\right|^{2}=$

$\sum_{j \geq 0} \sum_{l=-2 j}^{2 j-1} \mid \hat{\Psi}_{1}\left(\left.2^{-2 j} \xi_{1}\right|^{2}\left|\hat{\Psi}_{2}\left(2^{j} \frac{\xi_{2}}{\xi_{1}}-l\right)\right|^{2}=1\right.$

The collection of functions $\left\{\hat{\Psi}^{(0)}\left(\xi A_{0}^{-j} B_{0}^{-l}\right)\right\}$ constitute parts of $D_{0}$, as shown in Fig. 2.

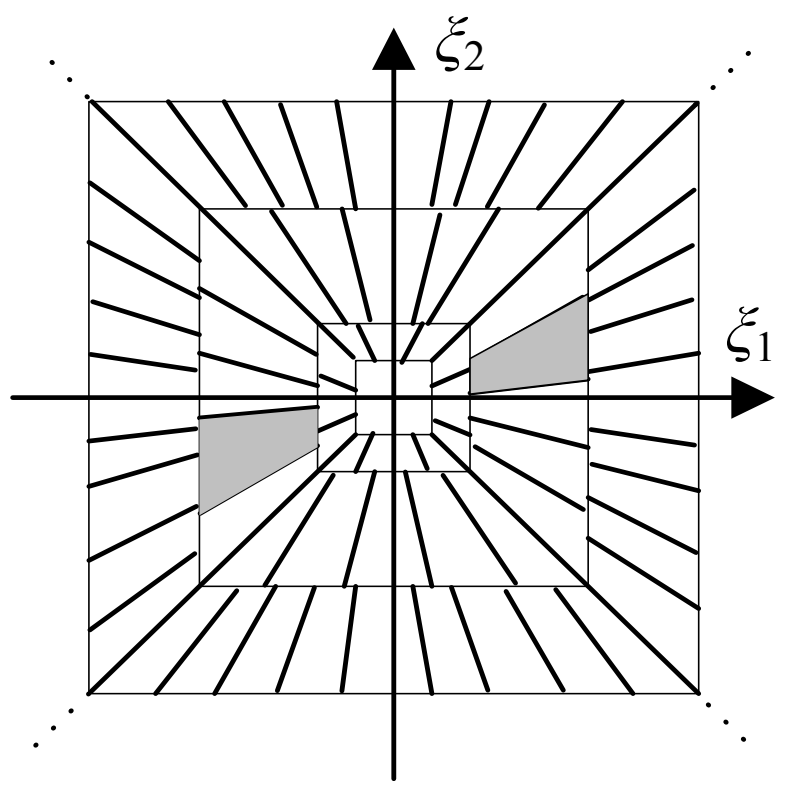

Fig. 2. Frequency tiling of the shearlet system
$D_{0}$ is supported in $[-1 / 2,1 / 2]^{2}$, thus,

$$
\begin{aligned}
& \left\{\hat{\Psi}_{j, l, k}^{(0)}=2^{3 j / 2} \Psi^{(0)}\left(B_{0}^{l} A_{0}^{j} x-k\right):\right. \\
& \left.j \geq 0,-2^{j} \leq l \leq 2^{j}-1, k \in Z^{2}\right\}
\end{aligned}
$$

forms a Parseval frame for function $L^{2}\left(D_{0}\right)^{\vee}=$ $\left\{f \in L^{2}\left(\mathbb{R}^{2}\right): \operatorname{supp} \hat{f} \subset D_{0}\right\}$.

\subsection{Fusion steps based on shearlet transform}

First, the geometrically registered source images need to be decomposed into a low-frequency sub-band $S_{c=0}(x, y)$, and a series of high-frequency sub-bands $S_{c, k}(x, y)$ by performing shearlet transform. With these, $c=1,2, \cdots, N$ represents the $c^{\text {th }}$ high-frequency sub-band of the image, $k=1,2, \cdots, M$ is the parameter for directions, whereas $(x, y)$ indicates the position of shearlets coefficients, by doing so, the multiscale and multi-directional decomposition on source images can be fulfilled. Next, the low frequency and high frequency coefficients are integrated with specific rules, respectively. Last, the fused image is produced by employing inverse shearlet transform. The detailed steps of image fusion under shearlets are as follows:

(a) Input: Registered source images $f_{1}$ and $f_{2}$.

(b) Decompose the original images into scale images as $T_{0}, T_{1}, \cdots, T_{N}$ by conducting non-subsampled pyramidal transform, where $T_{0}$ denotes the rough scale image, whereas $T_{1}, \cdots, T_{N}$ represent the subdivided images at scale level from 1 to $N$.

(c) Transform $T_{1}, \cdots, T_{N}$ to $F T_{1}, \cdots, F T_{N}$ using $2 \mathrm{D}$ fast Fourier transform (FFT) in frequency domain.

(d) Input $F T_{1}, \cdots, F T_{N}$ into the shear filter groups $S F_{c, k}$. The high-frequency coefficients $F_{c, k}$ and the highfrequency sub-band coefficients $S_{c, k}(x, y)$ are obtained using multi-directional decomposition and inverse $2 \mathrm{D}$ FFT, respectively.

(e) The low- and high-frequency coefficients of the fused image can be obtained by using the corresponding fusion rules.

(f) Output: Fused image is generated by employing inverse shearlet transform.

\subsection{Fusion course of the proposed method}

Given that $f_{1}$ and $f_{2}$ denote two source images, $F$ is the fused image. Fig. 3 shows the fusion process with fusion steps.

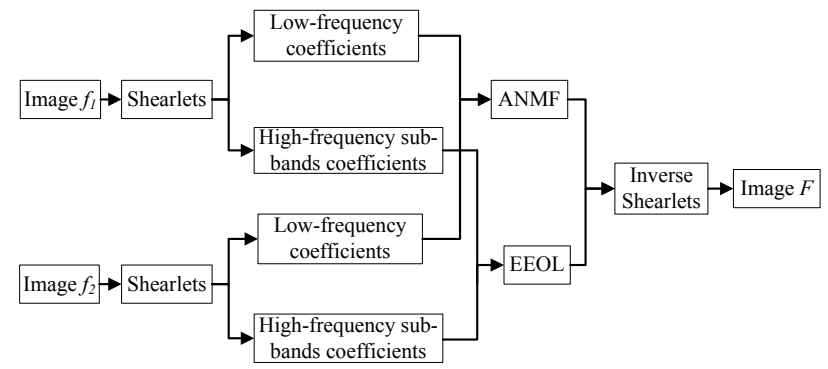

Fig. 3. Flowchart of fusion algorithm 
(1) Multiscale and multi-directional decompositions are conducted for source images $f_{1}$ and $f_{2}$ by adopting shearlet to obtain low-frequency sub-band coefficients $\left\{S_{i_{0}}^{f_{1}}(x, y), S_{i_{0}}^{f_{2}}(x, y)\right\} \quad$ and high-frequency sub-band coefficients $\left\{S_{i, l}^{f_{1}}(x, y), S_{i, l}^{f_{2}}(x, y)\right\}$.

(2) ANMF method [22] is used to achieve low-frequency sub-band coefficients $S_{i_{0}}^{F}(x, y)$, whereas EEOL is utilized to obtain high-frequency sub-band coefficients $S_{i, l}^{F}(x, y)$ for the fused image.

(3) Inverse shearlet transform is employed on the fused coefficients $S_{i_{0}}^{F}(x, y)$ and $S_{i, l}^{F}(x, y)$, which results in image $F$.

\subsection{Fusion rule for low-frequency sub-band coefficients}

In 1999, Lee and Seung proposed the non-negative matrix factorization (NMF), a novel method published in Nature [26]. In this theory, all elements are constrained to be nonnegative and only purely additive operations are permitted on components. Meanwhile, decomposed components are all required to be non-negative as well; consequently, the dimension of the original matrix is reduced. Generally, for a given non-negative matrix $V_{(n \times m)}$, NMF attempts to find two non-negative matrices $W_{(n \times r)}$ and $H_{(r \times m)}$, which satisfy $V \approx W H$.

To solve NMF problem, Euclidean distance and Kullback-Liebler divergence listed in Eqs. (7) and (8) are often used as objective functions.

$$
\begin{aligned}
& J(W, H)=\|V-W H\|^{2}=\sum_{i, j}\left[V_{i, j}-(W H)_{i, j}\right]^{2} \\
& J(W, H)=\sum_{i, j}^{n}\left[V_{i, j} \log \frac{V_{i, j}}{(W H)_{i, j}}-V_{i, j}+(W H)_{i, j}\right]
\end{aligned}
$$

Calculating the optimal values of $W$ and $H$ makes the reconstruction error between $V$ and $W H$ minimum; thus, solving ANMF can be cast into two optimization problems described as:

$$
\begin{aligned}
& H_{i j} \leftarrow H_{i j}+\alpha_{j} \eta_{i j}\left(W^{T} V-W^{T} W H\right)_{i j} \\
& W_{i j} \leftarrow W_{i j}+\beta_{j} \varsigma_{i j}\left(V H^{T}-W H H^{T}\right)_{i j}
\end{aligned}
$$

where $\eta_{i j}$ and $\varsigma_{i j}$ are small positive numbers, $\alpha_{j}(j=1,2, \cdots, n) \quad$ and $\quad \beta_{i}(i=1,2, \cdots, m) \quad$ are step-length parameters. As discussed in Reference [22], ANMF converges faster and can reach optimum with fewer iterations compared with the classical NMF.

The process of imaging acquisition often introduces various noises arising from sensors' nature or influences posed by the outside world; hence, an observed image is actually the composite of real image and noise. In ANMF algorithm, we can assume that $V=W H+\varepsilon$, where $\varepsilon$ denotes for the noise that will be convergent by using iterative updating rule; this description is inclined with the process of standard image fusion [27][28][29].

In ANMF theory, a part-based approximated representation of $W H$ from $V$ by iteration should be obtained, where columns of $W$ denote the value of feature basis $r$ and determines the dimension of feature subspace. Particularly, the implicit dimension of feature space for a given dataset is certain. In other words, when $r$ equals the actual dimension of the specific feature space, the basis of the feature space will be optimal. Therefore, when $r=1$, obtaining unique feature basis containing global features of the original data is possible.

Based on aforementioned scenarios, ANMF is appropriate for image fusion. Given that $f_{1}, f_{2}, \cdots, f_{k}$, which is acquired from $k$ multi-sensors with the size of $m \times n$ and denotes observed images, then matrix $V$ with the size of $m n \times k$ can be constructed by arranging the data from $f_{i}(i=1,2, \cdots, k)$ into $k$ column vectors in a row-by-row element-wise manner.As a result, matrix $V$, which contains $k$ column vectors $v_{1}, v_{2}, \cdots, v_{k}$, represent each of the observations with the corresponding column. Let

$\left\{\begin{aligned} v_{i}= & {\left[f_{11}^{i}, f_{12}^{i}, \cdots, f_{1 n}^{i}, f_{21}^{i}, f_{22}^{i}, \cdots, f_{2 n}^{i}, \cdots\right.} \\ & \left.f_{m 1}^{i}, f_{m 2}^{i}, \cdots, f_{m n}^{i}\right]^{T} \\ V= & {\left[v_{1}=f_{1}, v_{2}=f_{2}, \cdots, v_{k}=f_{k}\right] }\end{aligned}\right.$

The employment of ANMF on matrix $V$ with $r=1$ obtains a unique feature basis $W$. And $W$ contains holistic features of $k$ images. When $W$ is restored to the pixel-level of an original image, a new image is obtained, which has better quality than any individual modality source image.

\subsection{Fusion rule for high-frequency sub-band coefficients}

After shearlets decomposition, the directional sub-band coefficients exhibit detailed features of high-frequency components with respect to edge and texture. The fusion rule for high-frequency coefficients plays an important role in medical image fusion due to its clinical application. Moreover, contents of fused image must be inclined with those of source images as accurately as possible without any detail distortion that can cause clinical misjudgment. On the other hand, the fused image should be applicable for human eyes to identify the detailed texture, thereby achieving accurate diagnosis on lesions. Hence, the EEOL is adopted in high-frequency coefficient fusion.

The energy of Laplace (EOL) reflects the local definition of an image. A greater EOL value results in clearer image. According to Reference [30], EOL is superior when evaluating image quality compared with classical criteria. In EOL, the Laplacian operator is used, and the location distribution of a given pixel and the eight adjacent neighbors of the pixel are fully considered. The traditional EOL is defined as follows:

$$
\begin{aligned}
& E O L=\sum_{x} \sum_{y}\left(L_{x x}+L_{y y}\right)^{2} \\
& L_{x x}+L_{y y}=-L(x-1, y-1)-4 L(x-1, y) \\
& -L(x-1, y+1)-4 L(x, y-1)+20 L(x, y) \\
& -4 L(x, y+1)-L(x+1, y-1) \\
& -4 L(x+1, y)-L(x+1, y+1)
\end{aligned}
$$

where $L(x, y)$ is the pixel level at $(x, y)$. 
To enhance the correlation between diagonal pixels, and to avoid mutual interference posed by information from different orientations, EEOL is adopted, which is defined as:

$$
\operatorname{EEOL}(x, y)=\sum_{a} \sum_{b} W(a, b)(\operatorname{ISOL}(x+a, y+b))^{2}
$$

where $W(a, b)$ denotes the window function with the size of $3 \times 3$ and suffices the normalized feature $\sum_{a} \sum_{b} W(a, b)=1$ to enhance the functions of the pixel at the center of the window, as well as the related pixels along the edges. $W(a, b)$ is set as $1 / 16[1,2,1 ; 2,4,2 ; 1,2,1]$. Moreover, ISOL is the improved sum of Laplace expressed as:

$$
\begin{aligned}
& \operatorname{ISOL}(x, y)=|8 L(x, y)-4 L(x-1, y)-4 L(x+1, y)| \\
& +|8 L(x, y)-4 L(x, y-1)-4 L(x, y+1)| \\
& +|2 L(x, y)-L(x-1, y+1)-L(x+1, y-1)| \\
& +|2 L(x, y)-L(x-1, y-1)-L(x+1, y+1)|
\end{aligned}
$$

Then, the fusion rule for high-frequency directional subband coefficients is defined as:

$$
S_{c, k}^{F}(x, y)=\left\{\begin{array}{l}
S_{c, k}^{f_{1}}(x, y) \text { if } E_{E O L_{f_{1}}}(x, y)>E_{E O L_{f_{2}}}(x, y) \\
S_{c, k}^{f_{2}}(x, y) \text { else }
\end{array}\right.
$$

where $S_{c, k}^{f_{1}}(x, y)$ and $S_{c, k}^{f_{2}}(x, y)$ are the $c^{\text {th }}$ sub-band coefficients of the source images $f_{1}$ and $f_{2}$ in direction $k$, respectively. $\operatorname{EEOL}_{f_{1}}(x, y)$ and $E_{E O L}(x, y)$ denote the EEOL of $f_{1}$ and $f_{2}$ at $(x, y)$, respectively.

\section{Result analysis and discussion}

To verify the effectiveness of the proposed fusion method, two sets of strictly registered medical images are selected for fusion experiments. For comparison, PCA, Laplacian pyramid (LP), discrete WT (DWT), NSCT-based methods, and the proposed "ANMF+EEOL" method are adopted in Experiment 1, whereas intensity-hue-saturation (IHS)-based method and the shearlet-based "PCNN+Average", "NMF+neighborhood homogeneous measurement (NHM)" [22], and the proposed "ANMF+EEOL" methods are adopted in Experiment 2. All experiments are performed under the platform of AMD Athlon ${ }^{\mathrm{TM}} 64 \mathrm{X} 2$ processor 2.61 GHz with 2 GB RAM and Matlab R2010b.

In Experiment 1, two medical CT and MRI images are used with sizes $256 \times 256$. The $\mathrm{CT}$ image is clear in anatomical structure showing and is sensitive to calcification but has poor contrast in soft tissue demonstration. The intensity of the MRI image is directly proportional to the amount of proton. Normal soft tissues, such as muscle, fat, white matter, and gray matter, can be clearly identified in the MRI image. In addition, tumors or other lesions can also be well-distinguished. In clinical practice, the fused image of $\mathrm{CT}$ and MRI functions complementarily and is beneficial to either of the single modality in medical diagnosis and treatment.

The decomposition level for LP is 4 . The basis for DWT is selected as "haar" and its decomposition level is 3. For NSCT, the scaling and directional filters are set as "9-7" and "pkva", respectively; its decomposition level is 3 , and its direction levels are 4, 3, and 2. For DWT and NSCT methods, the fusion rules for low- and high-frequency coefficients are selected as "average" and "maximum", respectively. For the proposed method, the decomposition level of the shearlets is 3 , the direction level in subdivision scale is set as 6 , and the window size is $3 \times 3$.

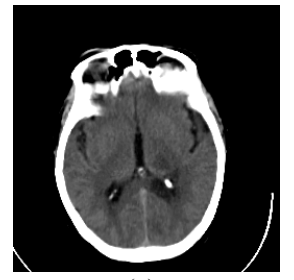

(a)

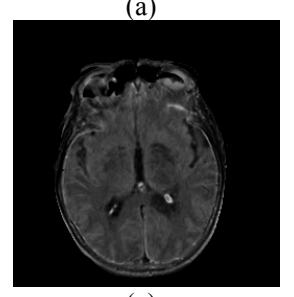

(c)

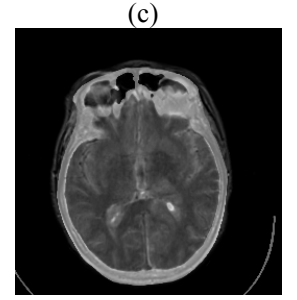

(e)

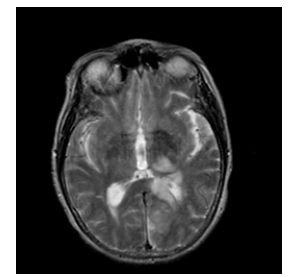

(b)

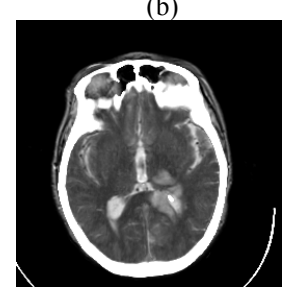

(d)

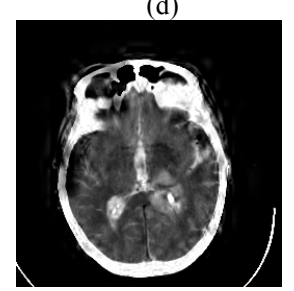

(f)

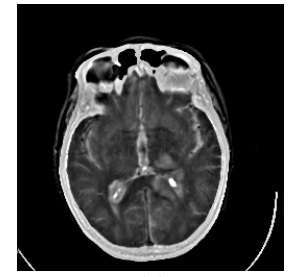

(g)

Fig. 4. Fusion results of five different methods. (a) CT image, (b) MRI image, (c) Fused image based on PCA, (d) Fused image based on LP, (e) Fused image based on DWT, (f) Fused images based on NSCT, (g) Fused image based on the proposed method.

Figs. 4(a) and 4(b) show the CT and MRI images, and Figs. 4(c)-4(g) show the fused images produced by PCA, LP, DWT, NSCT, and the proposed method, respectively. As shown from the fusion result in Fig. 4, all fused methods increase the amount of information but present a certain amount of differences. For example, PCA is a statisticsbased method that produces poor intensity; thus, Fig. 4(c) looks dim. Meanwhile, the other four approaches perform better in this aspect. In Fig. 4(d), the visual effect of textures for bone and soft tissue region is not as good as that of Fig. 4(g). Figs. 4(e) and 4(f) introduce false information with obvious blurring effects and distortion along the margins of the images. Additionally, the pseudo-Gibbs phenomenon occurred in Fig. 4(f); however, the image looks brighter than those produced by DWT and the proposed method. From visual point of view, the amount of information of the image produced by NSCT is sufficient, and artifacts at the middleleft portion in Fig. 4(f) can be easily found. Overall, the image produced by the proposed method has a natural tradeoff in brightness and contrast with added details transferred from source images. 
Aside from subjective evaluation, objective criteria are also explored. Information entropy (IE), mutual information (MI), average grades (AG), spatial frequency (SF), standard deviation (SD), and time cost are selected in this context. Table 1 shows the objective evaluation results obtained from the fused images in Experiment 1, with optimal value highlighted in bold. From Table 1, the proposed method has advantages over the other approaches in IE, MI, and SF. The largest IE indicates that the proposed method has the most information obtained with the best fusion. MI is used to measure the correlation between the source and fused images; a larger MI indicates more information obtained from the source image. Thus, Fig. $4(\mathrm{~g})$ is further correlated to source images compared with fused images produced by other methods. SF expresses the degree of activity for detail variation in spatial domain; a larger SF obtains better fused image. AG denotes the degree of the acquired detail and definition of the image; a larger AG results in better effect. The proposed method is slightly inferior to DWT. SD indicates the degree of deviation between the gray level of a pixel and the average of the fused image; richer detail variation creates larger SD with better image quality. From the above analysis, Fig. 4(g) achieves the best quality in three of five criteria; $\mathrm{AG}$ and $\mathrm{SD}$ are in the second place. Therefore, the statistics in Table 1 are inclined with the visual effects of Fig. 4. For time consumption, DWT does not have computational burden for multi-directional decomposition; thus, it takes the least time. However, the time cost of the proposed method decreased by $29.21 \%$ compared with the multiscale-based NSCT.

Table 1. Performance comparison of five different fusion methods

\begin{tabular}{c|l|l|l|l|c}
\hline & \multicolumn{1}{|c|}{ PCA } & \multicolumn{1}{c|}{ LP } & DWT & NSCT & Proposed method \\
\hline IE & 4.190 & 6.016 & 5.476 & 6.132 & $\mathbf{6 . 2 0 3}$ \\
MI & 3.861 & 3.080 & 2.916 & 5.386 & $\mathbf{5 . 3 9 0}$ \\
AG & 0.032 & 0.054 & $\mathbf{0 . 0 5 4}$ & 0.053 & 0.054 \\
SF & 16.425 & 19.671 & 18.624 & 18.130 & $\mathbf{1 9 . 8 7 6}$ \\
SD & 1.152 & 1.415 & 1.326 & $\mathbf{1 . 7 5 8}$ & 1.453 \\
Time cost (s) & 26.584 & 25.842 & $\mathbf{1 8 . 4 7 5}$ & 120.536 & 85.325 \\
\hline
\end{tabular}

In Experiment 2, four fusion methods are used, namely, the IHS method and the shearlet-based "PCNN+Average", "NMF+NHM", and the proposed "ANMF+EEOL" methods. Fig. 5(a) is the T1-weighted image. Fig. 5(b) is the PET image, which is mainly explored to detect the functional and metabolic changes of a disease. Theoretically, PET image can diagnose the cause of a disease earlier compared with CT and MRI images. Fig. 5(c) is the image based on IHS. Figs. 5 (d)-5(f) are the fused images produced by the shearlet-based "PCNN+Average", "NMF+NHM", and the proposed methods, respectively. Visually, the four fusion methods can extract features of source images and eject features into the fused images. Fig. 5(c) is comparable with water washed; thus, IHS-based method is inferior to other methods. The three shearlet-based methods resemble each other in overall sensation, with differences mainly reflected in high-frequency detail presentation. Specifically, "Average" is selected in high-frequency fusion; thus, it is the cerebral cortex in Fig. 5(d) is difficult to identify. Likewise, the "NHM" rule is applied to measure the similarity of corresponding neighborhoods for images to generate Fig. 5 (e). Therefore, the caudate nucleus in Fig. 5(e) is clearer compared with that of other figures; however, the texture of the cortex in Fig. 5(e) is unclear compared with that of Fig. 5(f). Therefore, the visual effect of the fused image created by the proposed method outperforms that of the other images.
Moreover, Fig. 5(f) has richer information and possesses higher contrast.

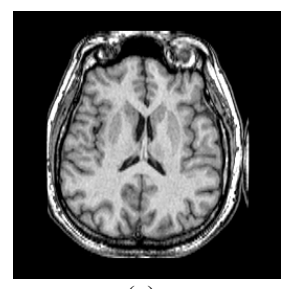

(a)

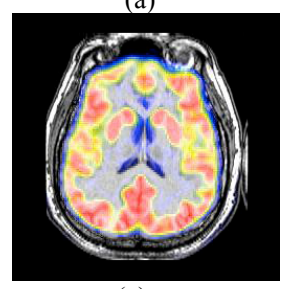

(c)

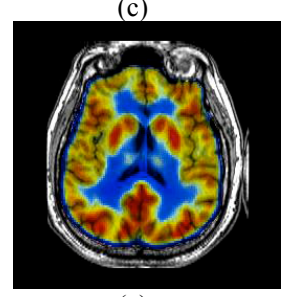

(e)

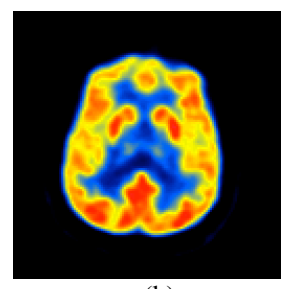

(b)

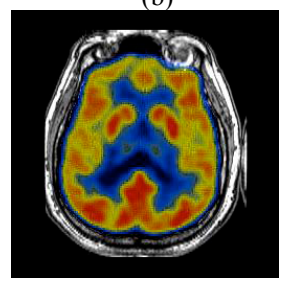

(d)

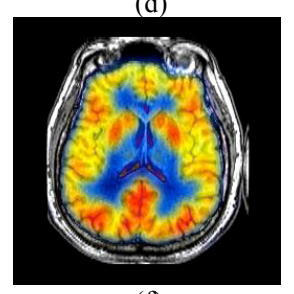

(f)
Fig. 5. Fusion results of IHS and three shearlet-related methods. (a) MRI image, (b) PET image, (c) Fused image based on IHS, (d) Fused image based on "PCNN+Average", (e) Fused image based on "NMF+NHM", (f) Fused image based on the proposed method.

Table 2. Performance comparison of IHS and three shearletrelated fusion methods

\begin{tabular}{c|l|c|c|c}
\hline & \multirow{2}{*}{ IHS } & \multicolumn{3}{|c}{ Shearlets } \\
\cline { 3 - 5 } & & PCNN+Average & NMF+NHM & Proposed method \\
\hline IE & 16.154 & 16.271 & 16.310 & $\mathbf{1 6 . 3 3 8}$ \\
MI & 6.487 & 6.725 & 6.738 & $\mathbf{6 . 8 6 8}$ \\
AG & 0.064 & 0.069 & 0.067 & $\mathbf{0 . 0 7 1}$ \\
SF & 20.087 & 21.688 & $\mathbf{2 1 . 8 4 5}$ & 21.761 \\
SD & 1.805 & 1.825 & 1.832 & $\mathbf{1 . 8 4 1}$ \\
Time cost (s) & $\mathbf{4 2 . 5 7 8}$ & 178.342 & 122.165 & 92.176 \\
\hline
\end{tabular}

A quantitative data analysis is also performed in this study. Table 2 lists the objective evaluation data of Experiment 2. From the table, the proposed method achieves the optimal value in IE, MI, AG, and SD but is only second best in SF. From objective evaluation, the proposed method works well and is consistent with the visual effect of the image in Fig. 5(f) for the MRI and PET fusion experiment. In terms of time consumption, the proposed method decreased by $48.32 \%$ and $24.55 \%$ when compared with the methods based on "PCNN+Average" and "NMF+NHM", respectively.

\section{Conclusions}

To improve image quality and optimize run time of the algorithm in medical image fusion, shearlet transform was used as the research object, and a model based on "ANMF+EEOL" fusion rule was developed to evaluate the validity of the proposed method. Comparisons were conducted between the traditional and proposed methods. The main conclusions are drawn as follows:

1) As a promising application in image processing, medical image fusion has been applied in clinical practice in recent years. Tens of fusion frameworks have been applied 
to meet the needs of clinical diagnosis and treatment, which exhibit the great potential of shearlets.

2) Fusion rules, such as "Maximum", "Minimum", and "Averaging" cannot accurately reflect details of image when used in medical image fusion; consequently, great uncertainty may occur in diagnosis. Moreover, employing PCNN- or NSCT-based methods intensifies computational complexity, which is adverse to the development of realtime clinical operation. The proposed "ANMF+EEOL" fusion method reduces the time consumed in operation without losing detail. Therefore, the proposed method is superior to most space-domain-based methods and traditional transform-domain-based methods.

3) The proposed method is superior to the selected multiscale fusion methods in subjective evaluation and objective criteria. Therefore, the proposed method is effective in CT-MRI and MRI-PET image fusion experiments. This method produces image with shorter time compared with the traditional transform-domain-based methods; thus, the proposed method is applicable as clinical algorithm in medical image fusion.
With proven superiorities of the proposed method in image quality and time-saving improvement, the fusion method based on "ANMF+EEOL" rule under shearlet domain is proposed. Until now, the proposed method has not been applied to images with other modalities nor has been used in actual clinical practices. In most cases, clinical fusion is influenced by factors of imaging quality, registration level, and denoising rate. Likewise, the proposed method is limited to considering the ideal status; therefore, the influencing factors must be introduced in developing clinical fusion system for future work.

\section{Acknowledgements}

This work was supported by the Project for Doctoral Development of China West Normal University under grant number "15E014".

This is an Open Access article distributed under the terms of the Creative Commons Attribution Licence

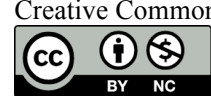

\section{References}

1. James, A. P., Dasarathy, B. V., "Medical image fusion: A survey of the state of the art". Information Fusion, 19(3), 2014, pp. 4-19.

2. Twycross, J., Aickelin, U., "Information fusion in the immune system". Information Fusion, 11(1), 2010, pp. 35-44.

3. Bhatnagar, G., Wu, Q. M. J., Liu, Z., "Directive contrast based multimodal medical image fusion in NSCT domain". IEEE Transactions on Multimedia, 15(5), 2013, pp. 1014-1024.

4. Geng, P., Wang, Z., Zhang, Z., "Image fusion by pulse couple neural network with shearlets". Optical Engineering, 51(6), 2012, pp. 1-7.

5. Dasarathy, B. V., "Information fusion in the realm of medical applications - A bibliographic glimpse at its growing appeal". Information Fusion, 13(1), 2012, pp. 1-9.

6. Singh, R., Khare, A., "Fusion of multimodal medical images using Daubechies complex wavelet transform-A multiresolution approach". Information Fusion, 19(3), 2014, pp. 49-60.

7. Xiao, B. Q., Jing, W. Y., Hong, Z. X., "Image fusion algorithm based on spatial frequency-motivated pulse coupled neural networks in nonsubsampled contourlet transform domain". Acta Automatica Sinica, 34(12), 2008, pp. 1508-1514.

8. Sapkal, R. J., Kulkarni, S. M., "Image fusion based on wavelet transform for medical application". International Journal of Engineering Research and Applications, 2(5), 2012, pp. 624-627.

9. Darwish, S. M., "Multi-level fuzzy contourlet-based image fusion for medical applications". IET Image Processing, 7(7), 2013, pp. 694700 .

10. Yang, L., Guo, B. L., Ni, W., "Multimodality medical image fusion based on multiscale geometric analysis of contourlet transform". Neurocomputing, 72(1), 2008, pp. 203-211.

11. Song, H., Yu, S., Song, L., "Fusion of multispectral and panchromatic satellite images based on contourlet transform and local average gradient". Optical Engineering, 46(2), 2007, pp. 1-3.

12. Bhateja, V., Patel, H., Krishn, A., "Multimodal medical image sensor fusion framework using cascade of wavelet and contourlet transform domains". IEEE Sensors Journal, 15(12), 2015, pp. 67836790.

13. Ganasala, P., Kumar, V., "CT and MR image fusion scheme in nonsubsampled contourlet transform domain". Journal of Digital Imaging, 27(3), 2014, pp. 407-418.

14. Li, T., Wang, Y., "Biological image fusion using a NSCT based variable-weight method". Information Fusion, 12(2), 2011, pp. 8592.

15. Guo, K., Labate, D., Anaral, L. A. N., "Optimally sparse multidimensional representations using shearlets". SIAM Journal on Mathematical Analysis, 39(1), 2008, pp. 298-318.

16. Lim, W. Q., "The discrete shearlets transform: A new directional transform and compactly supported shearlets frames". IEEE Transactions on Image Processing, 19(5), 2010, pp. 1166-1180.
17. Guo, R. G., Lu, P. X., Dong, Z. F., "Multi-focus image fusion based on non-subsampled shearlets transform". IET Image Processing, 7(6), 2013, pp. 633-639.

18. Liu, X., Zhou, Y., Wang, J., "Image fusion based on shearlets transform and regional features". AEU-International Journal of Electronics and Communications, 68(6), 2014, pp. 471-477.

19. Bhadauria, H. S., Dewal, M. L., "Medical image denoising using adaptive fusion of curvelet transform and total variation". Computers \& Electrical Engineering, 39(5), 2013, pp. 1451-1460.

20. Dong, L., Yang, Q., Wu, H., "High quality multi-spectral and panchromatic image fusion technologies based on curvelet transform". Neurocomputing, 159 (C), 2015, pp. 268-274.

21. Wang, J., Lai, S. Y., Borjer, T. H., "Image fusion based on WRV and NHM rules for medical digital image in contourlet domain". Journal of Digital Information Management, 14(3), 2016, pp. 151159.

22. Wang, J., Lai, S. Y., Li, M. D., "Improved image fusion method based on NSCT and accelerated NMF". Sensors, 12(5), 2012, pp. 5872-5887.

23. Zhou, S., Shi, J., Zhu, J., "Shearlets-based texture feature extraction for classification of breast tumor in ultrasound image". Biomedical Signal Processing and Control, 8(6), 2013, pp. 688-696.

24. Wang, L., Li, B., Tian, L. F., "Multi-modal medical image fusion using the inter-scale and intra-scale dependencies between image shiftinvariant shearlets coefficients". Information Fusion, 19(1), 2014, pp. 20-28.

25. Candes, E. J., Donoho, D. L., "Continuous curvelet transform: I. Resolution of the wavefront set". Applied and Computational Harmonic Analysis, 19(2), 2005, pp. 162-197.

26. Lee, D. D., Seung, H. S., "Learning the parts of objects by nonnegative matrix factorization". Nature, 401(6755), 1999, pp. 788791.

27. Yokoya, N., Yairi, T., Iwasaki, A., "Coupled nonnegative matrix factorization unmixing for hyperspectral and multispectral data fusion". IEEE Transactions on Geoscience and Remote Sensing, 50(2), 2012, pp. 528-537.

28. Wang, J., Wang, H. J., Li, Y. H., "Image fusion and evaluation of geological disaster based on remote sensing". International Journal of Online Engineering, 10(4), 2014, pp. 28-34.

29. Zeng, K., Yu, J., Li, C., "Image clustering by hyper-graph regularized non-negative matrix factorization". Neurocomputing, 138(11), 2014, pp. 209-217.

30. Huang, W., Jing, Z., "Multi-focus image fusion using pulse coupled neural network". Pattern Recognition Letters, 28(9), 2007, pp. 11231132. 